\title{
Resource-saving technologies for cleaning gas emissions in the production of building materials
}

\author{
Tatiana Skorik ${ }^{1}$, Natalia Galkina ${ }^{1, *}$, and Elena Glazunova ${ }^{1}$ \\ ${ }^{1}$ DSTU, Rostov-on-don, Russia
}

\begin{abstract}
High requirements to ensure sanitary and hygienic working conditions and reduce harmful emissions into the environment are provided through ventilation systems equipped with efficient and economical equipment. The task of improving the air basin is to reduce the amount of harmful substances in the ambient air to the level of MPC and lower, which, with increasing volumes of ventilation and process emissions, determines the increased requirements for industrial filters. The most effective are wet devices, in which the process of gas cleaning is carried out through interaction with the liquid, most often with water. The authors developed a number of high-efficiency dust-collecting apparatuses, in which the majority of methods for contacting elements with a liquid are realized simultaneously, which determines the maximum completeness of gas purification from dusts with different physico-chemical properties. One of them is an aerohydrocyclone intended for combined simultaneous cleaning of a technological product in the form of a suspension and dusty air removed from the shelters of technological equipment. This apparatus with internal circulation of liquid, where the concentration of trapped dust in the irrigating liquid affects the efficiency of dust collection, the frequency of water replacement, the operating mode and the load of the water-slurry farm. The particulate materials of the slurry intensifies the process of dispersion, irrigating liquid and its structural viscosity, as well as the stability of the formed foam, which contributes to a more effective manifestation of various wet dust collection mechanisms. The implementation of the installation of a wet dust collector for complex cleaning in the technological line for the preparation of lime milk from the construction materials factory made it possible to reduce the concentration of dust in emissions at the maximum level of ensuring the economy of aspiration systems. The obtained results testify to the positive experience of the operation of wet dust collectors for complex cleaning in the building materials industry, and the regularities connecting the operation modes of the apparatus with the properties of trapped dusts and the features of technological processes make it possible to recommend them for use in various enterprises.
\end{abstract}

* Corresponding author: natash-galkina@mail.ru 
The growing anthropogenic impact on the environment determines the growing importance of the problem of environmental protection and possible impacts associated with manufactured and consumed products, increases the interest in developing methods aimed at reducing these effects of production. One of the ways to minimize the negative consequences of industrial production, including when manufacturing construction products is the use of the concept of product life cycle (PLC) [1]. Assessment of the life cycle of products analyzes the use of natural resources and the environmental consequences of their release in the production of products as a sequence of different selective stages of the process that function individually, but which have mutual influence and determine the quality of construction.

Environmental damage to the potential environmental impact from the receipt and use of resources, and their release in the manufacture of products, their use and as a result of disposal depends on the effectiveness of each stage [4]. Production systems consist of a number of single processes, interconnected by intermediary product flows and waste from processing with other production systems and with the environment. Modern industrial production places high demands on ensuring sanitary and hygienic working conditions, creating the required microclimate parameters for carrying out technological processes, and reducing harmful emissions to the environment. These issues are solved with the help of ventilation systems equipped with efficient and economical equipment. The increase in the amount of work and the costs of organizing ventilation is explained by the development of technological processes and the emergence of new technological equipment, an increase in the role of comfort in the environment of people's stay, tightening of regulatory requirements for the production and the environment. The main goal of improving the air basin is to reduce the amount of harmful substances in the ambient air to the level of PEL and lower [4], which, with increasing volumes of ventilation and process emissions, determines the increased requirements for industrial filters.

It is known that one of the most effective are wet devices, in which the process of gas purification is carried out due to the interaction with the liquid, most often with water. The necessary contact of suspended particles with it can be provided in various ways: by passing a gas through a layer of liquid, in contact with a film of a draining liquid, by interacting with droplets of dispersed liquid, in a foam layer, etc. A promising direction is the creation of apparatuses combining different physical mechanisms for trapping, which makes it possible to increase the total degree of gas purification $[5,6,8]$. The authors developed a number of high-efficiency dust-collecting apparatuses, in which the majority of methods for contacting particles with a liquid are realized simultaneously, which determines the maximum complete purification of gases from dusts with different physicochemical properties $[9,10]$.

An example of such a device is an aerohydrocyclone (patent No. 54538) [3].

This device is designed for combined simultaneous cleaning of a technological product in the form of a suspension and dusty air, removed from the shelters of technological equipment. The use of a comprehensive purification scheme allows to achieve an increase in efficiency, economy, and a decrease in the energy intensity of the technological process.

The hydro-cyclone includes a body, a dust-gas injection unit, a clarified slurry collection unit, a droplet separation unit, and at the same time has an internal volume increased by $15-25 \%$. In the upper cylindrical part there is provided a tangential connection for the introduction of a dusty gas, which is in the form of a venturi with a central supply of a refluxing suspension, and a branch pipe for removing the clarified suspension is installed in the lower conical part, moving vertically along the axis of the apparatus and a branch for draining the slurry. The upper part of the aerohydrocyclone is made with a rupture of the exhaust pipe and a device for the separation of droplets. 
Such a design of the apparatus allows, by increasing the volume of the housing, to improve the separation of the liquid and gaseous phases and to reduce the droplet. Displacement of the nozzle for introducing dusty air into the housing and placing the droplet separator inside the housing makes the device more compact and reduces useless pressure losses.

The inlet part of the dusty air, intended simultaneously for mixing it with the irrigating slurry, is similar to the Venturi pipe: the confuser is the transition from the duct to the inlet branch pipe with the nozzle placed in it - the sprayer of the supply line of the technological suspension; throat - the annular gap between the walls of the body and the exhaust pipe. The part of the body from the cutoff of the exhaust pipe to the surface of the suspension serves, like the diffuser, for the coagulation and separation of droplets and dust particles, and these processes proceed much more efficiently due to intense turbulence and the presence of centrifugal forces. In the conical part of the body, under the influence of intensive centrifugal separation, the suspension is clarified and the size of the boundary grain is classified according to the height of its discharge. At the bottom of the conical part, a branch pipe for draining the clarified suspension is installed, which can be moved along the vertical axis by means of the guide bushings. The possibility of vertical movement of the drainage pipe, provided by the design of the apparatus, provides adjustment of the size and concentration of particles of the solid suspension according to technological requirements. The slurry separated from the slurry is removed through the discharge pipe.

The design of the device and its operating mode provides specific irrigation of dusty air from 10.0 to $12.51 / \mathrm{m} 3$, which is an order of magnitude greater than the usual values in Venturi scrubbers. This, as well as high turbulence, a film of liquid on the walls of the body 1 , an open funnel-shaped water surface separated from the air, but retaining the rotational motion of the suspension, causes a high efficiency of air purification from dust.

As the aerohydrocyclone refers to apparatus with internal circulation of liquid, it is important to take into account the influence of the concentration of trapped dust in the irrigating liquid on the efficiency of dust collection, and consequently, the frequency of water replacement, the operating regime and the load of the water-slurry farm.

As studies have shown, the presence of suspended matter in the wetting liquid of wet dust collectors to certain limits contributes to an increase in the degree of purification. For aerohydrocyclone, the maximum increase in the efficiency of dust collection takes place at a slurry concentration of up to $15 \%$ [7].

Most dusts are inactive and hydrophilic, which makes it possible to use in wet dust collectors to irrigate their suspensions. The particulate matter of the slurry intensifies the process of dispersion, irrigating liquid and its structural viscosity, as well as the stability of the formed foam, which contributes to a more effective manifestation of various wet dust collection mechanisms. In Fig. 1 depicts the dependence of the increase in the efficiency of dust collection on the concentration of suspended matter in the working liquid of wet dust collectors for various dusts of building materials.

An example of the use of wet dust collectors for complex cleaning is the installation of a wet centrifugal dust collector in the technological line for the preparation of lime milk [2] of the construction materials plant (CMP).

The main hazard, which is released in the process of lime production when it is repeatedly processed and transported, is lime dust $\left(\mathrm{PEL}=2 \mathrm{mg} / \mathrm{m}^{3}\right)$.

Sources of harmful substances that pollute the atmosphere are emissions of aspiration systems of a limestone warehouse with galleries, raw materials sorting departments, a furnace department, a crushed lime warehouse and technological equipment for the line for the preparation of lime milk. The total amount of emissions is $29800 \mathrm{~m}^{3} / \mathrm{h}$ with gross dust accumulations of $0.3854 \mathrm{t} / \mathrm{h}$. 
With the average operating efficiency of dust collection in a two-stage cleaning scheme with installation of cyclones ЦН-15 and filters СМЦ 90\% loss of lime was about 185 tons per year.

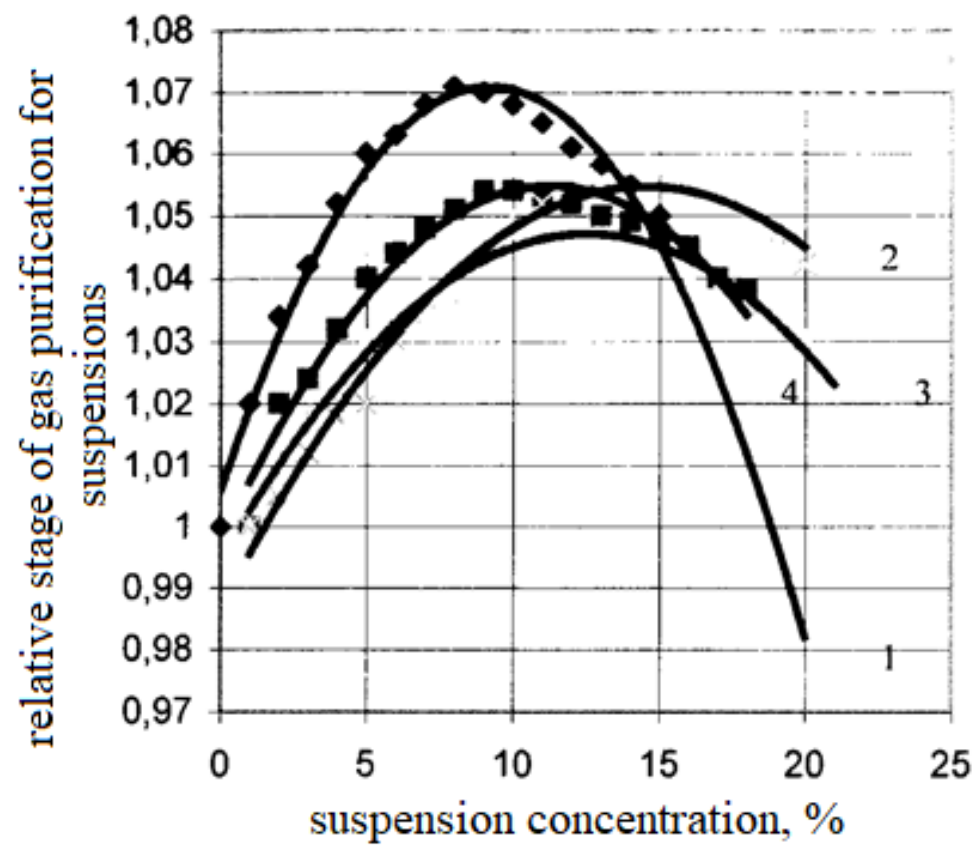

Fig. 1. Dependence of the degree of gas purification on the concentration of the irrigating suspension: 1-gypsum; 2-lime; 3-phenoplast; 4-cement.

In order to increase the efficiency and profitability of the dust collection systems of the lime shop, an aspiration unit with a wet dust collector for complex cleaning - an aerogicycle cyclone built into the production line - has been developed. It has the ability to work on a technological suspension of lime milk. Simultaneous impact on the three-phase environment of various physical mechanisms of interaction provides a high degree of purification, both aspiration air, and technological product.

The combination of several structural units that realize various mechanisms of contact of phases in one apparatus can eliminate a number of drawbacks inherent in wet dust collectors, for example, drip holes, the cost of water-slurry farming, and also increase specific irrigation of the gas to be cleaned and drastically reduce emissions of not only dust and harmful gases and vapors.

The introduction of the proposed purification scheme at various sites of lime production allowed to reduce the concentration of dust in emissions from an average of $12.39 \mathrm{~g} / \mathrm{m}^{3}$ to $0.05 \mathrm{~g} / \mathrm{m}^{3}$, with the maximum level of economic efficiency of aspiration systems.

The results of the production experiments show the advantages of the aspiration plant with the aerohydrocyclone in front of the standard hydrocyclone and Venturi scrubber, which consist of a reduction in capital and operating costs, an increase in the efficiency of aspiration air cleaning to $99.9 \%$, complete utilization of the trapped product, release of production areas and an increase of $9-11 \%$ of the utilization rate of technological equipment, reduction of power consumption to $0.7 \mathrm{~kW}$ per $1000 \mathrm{~m}^{3}$ of air [8]. 
The obtained results testify to the positive experience of the operation of wet dust collectors for complex cleaning in the building materials industry, and the patterns connecting the operation modes of the apparatus with the properties of the dusts trapped and the features of technological processes make it possible to recommend them for use in various enterprises [9].

\section{References}

1. GOST R ISO 14040-2010 Environmental management. Cycle assessment. Principles and structure

2. A method of complex purification of the technological product in the form of a suspension and aspiration air from the same solid impurities. Auth. UD. no. 893219, Byul. an image. № 48, 30.12.98

3. Aerohydroplane. Pat. 54538 OF THE RUSSIAN FEDERATION, IPC B04C 5, 12.12.2005

4. GOST R 54298-2010 Environmental management Systems. Procedure for certification of environmental management systems

5. GOST R ISO 20140-1-2014 Industrial automation systems and integration. Assessment of energy efficiency and other factors of production systems affecting the environmentISO 14004 Environmental management systems - General guidelines on principles, systems and support techniques

6. E. Shtokman, V. Shilov, E. Novgorod, T. Skorik, Ventilation, air conditioning and air purification in the food industry (M.: ASV, 2007, 668 p.)Industrial ventilation. 19 th Edition A Manual of Recommended Practice (ACGIH, Cincinnati, OH, USA, 1986)

7. T. Skorik, N. Strakhova, E. Glazunova, Design principles of dust collecting devices Science, 4 (13), 206 (2012)

8. E. Boguslavsky, E. Glazunov, Centrifugal wet dust collector. (NO. 1037933, MKI 01 D/10/N 2969708/23-26. Announced 08.08.80. Bul. no. 32. 08.08.80)

9. T. Skorik, A. Trubnikov, Science, 4 (2012) 